\title{
FUTURE DIRECTIONS IN THE STORAGE RING DEVELOPMENT FOR LIGHT SOURCES
}

\author{
Albin F. Wrulich, PSI, Switzerland
}

\begin{abstract}
Major performance objectives for storage-ring based light sources are small emittance and energy spread, high beam stability, and full exploitation of the photon energy from a few eV up to hard X-rays. Most of the recent- generation light sources are built up by highly optimized lattices of the Double Bend- or Triple Bend Achromat type. There is little room left to further optimize these lattices to even smaller emittances. Only a local adjustment of the optics at the source points might still bring some benefit. With decreasing emittance orbit stability is becoming more and more important. Cures for vibrations, thermal effects and ground motion have to start at the basic design of the building, the storage ring infrastructure and the magnet support system. Beam instabilities are especially harmful as they increase the energy spread and as a consequence deteriorate the higher harmonics of the undulator radiation. Well optimized beams suffer from lifetime problems due to Touschek scattering. Particular problems arise when short bunches are requested, for instance for storage ring based lasers. Medium-energy machines are able to produce high brilliance in the hard X-ray range by means of mini-undulators with small gaps. This, again, has an adverse effect on the beam lifetime due to beam gas scattering.

The current trend in storage ring development for future Light Sources is discussed. What are the crucial issues and where is improvement desirable and possible? Is there still room for improving the basic design of a light source? Are there alternatives to the 'classical' lattices which could be used for the next generation? Or is improvement possible only on the operational field?
\end{abstract}

\section{INTRODUCTION}

At first glance, storage-ring based light sources seem to have reached their performance limits. The optimization of the structure towards low emittance is very advanced and not much room is left for further improvements. An increase of the ring size is always an alternative, but beside the usual problem of budgetary constraints, large circumference machines working at low energies are suffering from adverse coherent effects and lifetime problems. At the last Workshop on 4th Generation Light Sources [1], an enthusiastic vote was given that linac driven SASA FELs will be the machines of the 4th generation. In this meeting and in subsequent publications [2] it was stated that they are fulfilling all the requirements for a next generation light source'! On the other hand, a pilot project demonstrating the SASA prin- ciple for X-rays is still missing and from there it would be still a long way to a user facility. Fundamental limits for the lasing process are reached at photon energies of 10$20 \mathrm{keV}$ [3]. Stability might also be an issue, and is better under control in storage-ring based sources. Last but not least, storage ring technology is extremely well developed [4] and we can still benefit from it in the future. It is therefore necessary to think also about the future of storage ring based light sources.

\section{PERFORMANCE GOALS}

At the Workshop on 4th Generation Light Sources [1] the key parameters for future sources were defined to be: brilliance, coherence, short bunch lengths, high photon energies ( up to $100 \mathrm{keV}$ ) and stability. A factor 100 in the reduction of the emittance was considered as a target goal which would allow the realization of a new class of experiments. Figure 1 shows the development of brilliance for light sources from the first generation to a possible 4th generation. A next step of a factor 100 would simply mean a reduction of the horizontal emittance to about $510^{-11}$ rad-m. As demonstrated in Figure 1, with each new generation also the vertical emittance was simultaneously reduced by about the same ratio. For the next generation, it might be advisable to not further reduce the vertical emittance in the same way. This would alleviate problems with Coulomb scattering inside the bunch. Furthermore, already now the vertical emittance is at the diffraction limit for $10 \mathrm{keV}$ photons.

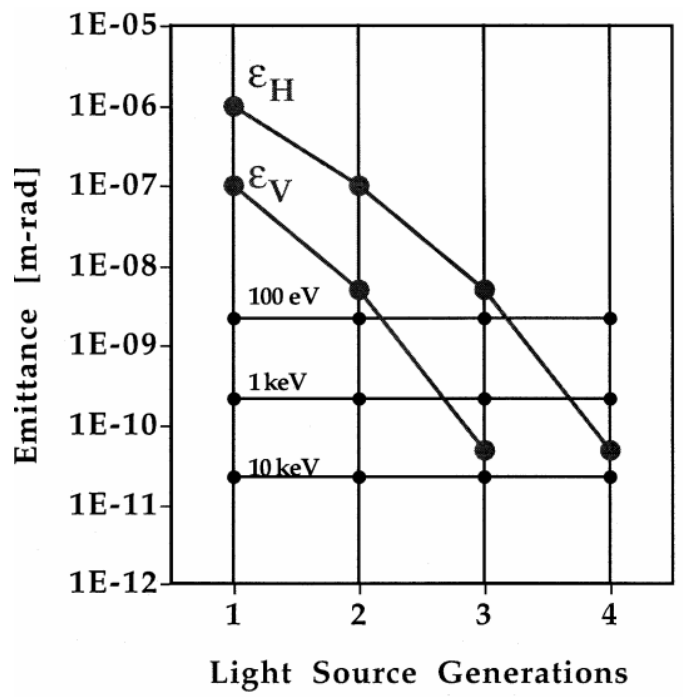

Fig. 1: Development of the brilliance in light sources with a possible future step for Fourth Generation Sources. 


\subsection{LOW EMITTANCE}

The emittance of a storage ring is solely defined by magnet structure and energy. It should be analyzed here, which further reduction we can expect for the 4th generation and what the solutions are for that. The 'classical' structures for light sources are DBA and TBA (Double and Triple Bend Achromat structures). Structures have been developed with more magnets per achromat, since for constant deflection of an achromat, the angle of the individual magnet is reduced and correspondingly the emittance which scales with the third power of the angle. Ultimately, such an expansion leads to a FODO structure with dispersion matching towards both ends. This, of course tends to increase the length of an achromat. As figure of merit for comparing structures with the same emittance we can take the ratio of straight section length to achromat length.

All these structures have well defined limits for the emittance. They are given by the individual limits for the single bending magnets, with dispersion suppression towards both ends and the dispersion symmetry points in the middle for the inside bending magnets. For DBA and TBA the theoretical limits $[5,6]$ are given by:

$$
\begin{aligned}
& \varepsilon_{H}=\frac{C_{q} \gamma^{3}}{J_{H}} \cdot K \cdot \phi_{B}^{3} \\
& \text { with } \quad K=\frac{1}{4 \sqrt{15}} \text { for } D B A \quad K=\frac{7}{36 \sqrt{15}} \text { for } T B A
\end{aligned}
$$

where $\gamma$ is the Lorentz factor, $\mathrm{J}_{\mathrm{H}}$ the horizontal damping partition number, $\phi_{\mathrm{B}}$ the deflection of one bending magnet, and $\mathrm{C}_{q}, \mathrm{~K}$ are numerical factors.

Intrinsic matching requirements reduce the numerical factor for the TBA limit to about the value of the DBA [7]. Nevertheless, there is still the third power of the bending angle ratio that considerably reduces the emittance for an TBA as compared to a DBA. On the other hand, whereas a TBA always requires a small dispersion in the center of the structure, a DBA allows an increase of the dispersion inside the achromat by expansion, which makes the chromatic compensation with sextupoles easier. This is the reason why large machines with low individual bending angle and correspondingly small dispersion are always built up by DBA structures. For the $3^{\text {rd }}$ generation sources, the ratio between actual emittance and minimum emittance is varying between 1.5-3 for DBAs and 2-4 for TBAs. Not much space is left for further reducing the emittance for these 'classical' structures, maybe a factor of two. The simplest way for reducing the emittance, as mentioned before, is to increase the number of bending magnets. Unfortunately, a machine with large circumference working at low energies reveals a couple of adverse effects which are difficult to handle. Keeping the energy fixed by increasing the length affects the energy loss per turn as $\sim 1 / \mathrm{L}$, damping time as $\sim \mathrm{L}^{2}$, energy spread as $\sim 1 / \mathrm{L}^{1 / 2}$ and emittance as $\sim 1 / \mathrm{L}^{3}$ Going to the maximum capability of such an expanded ring (i.e. with a bending magnet field as before the expansion) all the adverse effects would be eliminated and even be improved as compared to the original not expanded machine. In addition, the spectrum would be extended further into the high photon energy range. Actually, all existing machines actually are working at the maximum energy for these reasons. Another way of alleviating some of the adverse effects due to circumference increase and of further reducing the emittance is the introduction of damping wigglers in the structure. Storage ring relevant parameters would be modified [8] according to:

$$
\begin{aligned}
& \left(\frac{\sigma_{E}}{\sigma_{E o}}\right)^{2}=\frac{U_{o}}{U_{w}}\left[1+\frac{4}{3 \pi} \frac{L_{w}}{L_{o}}\left(\frac{\rho_{o}}{\rho_{w}}\right)^{3}\right] \\
& \frac{\varepsilon_{H_{F}}}{\varepsilon_{H_{o}}}=\frac{U_{o}}{U_{w}}\left[1+\frac{L_{w}}{L_{o}} \frac{H_{w}}{H_{o}}\left(\frac{\rho_{o}}{\rho_{w}}\right)^{3}\right] \\
& \frac{U_{w}}{U_{o}}=1+\frac{1}{2} \frac{L_{w}}{L_{o}}\left(\frac{\rho_{o}}{\rho_{w}}\right)^{2} \\
& H_{w}=\left(\frac{\lambda_{w}}{2 \pi \rho_{w}}\right)^{2} \cdot \frac{4\langle\beta\rangle}{15 \pi}
\end{aligned}
$$

$\mathrm{L}, \rho$, and $\mathrm{U}$ are total length, bending radius and energy loss per turn for wigglers (w) and bending magnets (o). $\mathrm{H}$ is the Courant Snyder invariant, $\lambda_{\mathrm{w}}$ the wiggler period length and $\langle\beta\rangle$ the average beta function in the wiggler section. As an example for an expanded structure, let us double the size of the APS [9] and use the additional 40 straight sections for the implementation of 5.2 meter long damping wigglers with 2 Tesla field and $10 \mathrm{~cm}$ period length. We would obtain a machine with the parameters listed in Table 1.

Table 1 Example for an 4th Generation Light Source ( 2 x APS)

\begin{tabular}{|l|l|}
\hline PARAMETERS & \\
\hline Energy, GeV & 7 \\
\hline Circumference, $\mathrm{m}$ & 2120 \\
\hline Natural emittance, m-rad & $5.410^{11}$ \\
\hline Energy spread & $6.810^{4}$ \\
\hline Damping times $\tau_{\mathrm{x}}, \tau_{\mathrm{y}}, \tau_{\mathrm{s}}, \mathrm{ms}$ & $4,4,2$ \\
\hline Momentum compaction & $610^{5}$ \\
\hline Number of straight sections & 80 \\
\hline Straight sections with damping wigglers & 40 \\
\hline Total damping wiggler length, $\mathrm{m}$ & 208 \\
\hline Wiggler field, $\mathrm{T}$ & 2 \\
\hline Wiggler period length, $\mathrm{m}$ & 0.1 \\
\hline
\end{tabular}


The emittance would provide diffraction-limited photons in the $10 \mathrm{keV}$ range and a high degree of coherence. The 'damping' wiggler would have interesting properties for a wide field of applications. Brilliance and horizontal flux density are shown in Figures 2 and 3 (for a beam current of $100 \mathrm{~mA}$ ). The period length could be optimized towards the experimental needs, as long as the emittance blow up due to the self dispersion is kept small. It must be notified that the power from such a wiggler would not be easy to handle ( $125 \mathrm{~kW}$ for $100 \mathrm{~mA})$ and a reduction of the current would presumably be necessary, which proportionally reduces the brilliance.

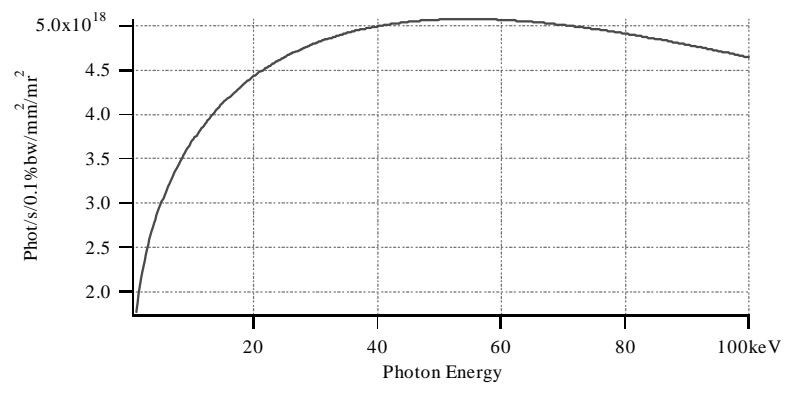

Fig. 2: Brilliance as a function of photon energy for the damping wiggler $(B=2 \mathrm{~T}, \lambda=0,1 \mathrm{~m}, \mathrm{~L}=5,2 \mathrm{~m})$.

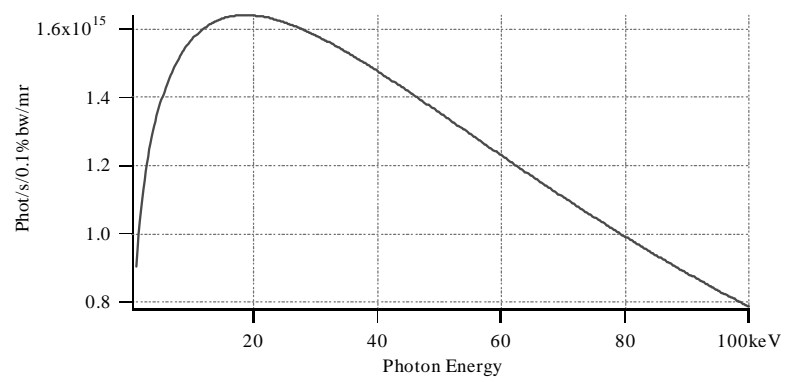

Fig. 3: Horizontal flux density as a function of photon energy for the damping wiggler.

\subsection{SHORT PULSES}

The direct method for achieving short light pulses is the reduction of the bunch length of the electron beam. Several methods have been discussed and tested in various places. The brute force method is to increase the RF-gradient, which according to the definition of the bunch length as:

Low current: $\sigma_{s} \approx\left\{\frac{\alpha_{c} \gamma^{3}}{\omega_{R F} V_{R F}}\right\}^{1 / 2}$ High current: $\sigma_{s} \approx\left\{\frac{\mathrm{I}\left|\frac{Z_{\mathrm{I}}}{n}\right|}{\omega_{R F} V_{R F}}\right\}^{1 / 3}$

can be done by increasing the RF-voltage $\left(\mathrm{V}_{\mathrm{RF}}\right)$ or the RFfrequency $\left(\omega_{\mathrm{RF}}\right)$. An increase of the voltage by adding more cavities is costly. A harmonic cavity working at a multiple of the fundamental frequency combines the advantages due to voltage increase and higher frequency in order to reach higher gradients. Pulsed RF would be an alternative, but provides only a low duty cycle. The same holds for a rotation of the bunch by additional cavities. A lot of hope was put in the reduction of the momentum compaction factor $\left(\alpha_{c}\right)$ in order to reach short bunch lengths. Experiments were performed at Lure, ALS, ESRF and others. Unfortunately, the outcome is not promising. Low and negative momentum compaction effectively reduces the bunch length at low currents. For the interesting range of higher currents, however the bunch length is independent of the momentum compaction. Even if the low intensity would be acceptable for the performance of certain experiments, it creates a conflict with users preferring high brilliance and correspondingly high currents. An alternative method has been developed by [10] which does not generate this compatibility problem. There, in an ID section, the electron beam is interacting with a femtosecond laser pulse. This creates a modulation of the energy corresponding to the pulse length of the laser beam. Subsequently, the light generated by the off energy fraction of the beam can be extracted by spectral or dispersive separation. In spite of this interesting approach, one must conclude that linac-based SASE FELs will be far superior in creating pulses in the 100 fs range.

\subsection{HIGH PHOTON ENERGIES}

The simplest way to reach higher photon energies is to spend more money, i.e. to increase the dimensions of the storage ring and simultaneously the energy. The question here is more what can be reached within certain budgetary boundary conditions or what is the cost- optimized machine for a certain photon energy range. The new upcoming generation of medium-energy machines, DIAMOND, SLS and SOLEIL is trimmed in this direction. SLS, currently under construction in Switzerland, is the first machine of the medium energy range which will enter the regime of hard X-rays with high brilliance. This is achieved by the use of mini-undulators and the utilization of higher harmonics of the spectrum. Both aspects of this approach are posing severe conditions on machine performance and machine operation. Low gaps adversely affect the beam gas scattering lifetime, which scales inversely proportional to the square of the gap size. But not only this, also the Touschek scattering lifetime can be reduced. For the SLS, a strong nonlinear coupling of the horizontally oscillating Touschek scattered particle transfers the motion into the vertical plane where it get lost at the low- gap insertion device aperture [11]. Only a careful coupling minimization can alleviate this effect. A variation of the Touschek lifetime with vertical aperture for the SLS is shown in Figure 4. 


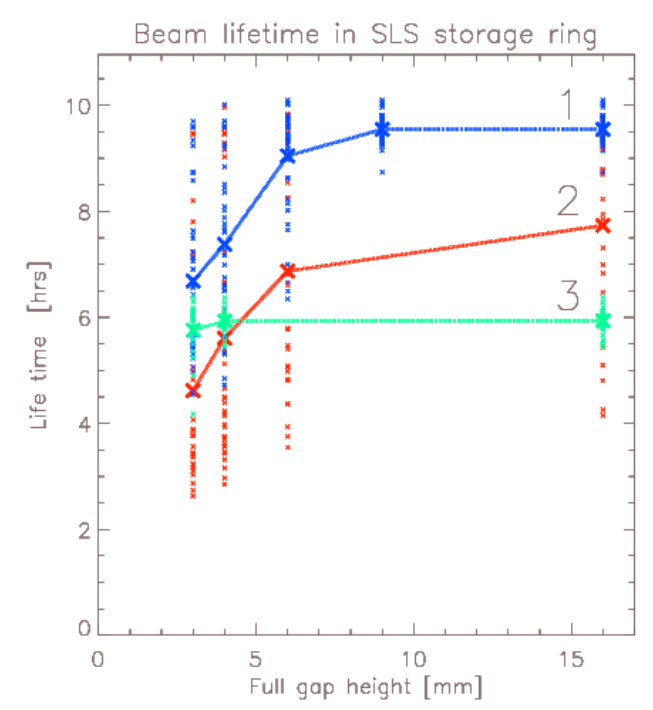

Fig. 4: Touschek lifetime as a function of vertical stay clear aperture in the undulator. The meanings of the different curves are: (1) tracking for constant energy deviation, $(2,3)$ tracking with synchrotron oscillations and (3) with additional coupling control. The emittance coupling is $2.8 \%$ or $(1,2)$ and $1 \%$ after minimization for $(3)$.

The effect will presumably be increased if more circularly polarized undulators are inserted into the storage ring structure, since their horizontal magnet field will transfer motion into the vertical plane. This demonstrates that increasing the momentum acceptance is not always the way to cure Touschek scattering life time reductions. Only a reduction of the scattering events, i.e. a reduction of particle density, as reached with a bunch lengthening cavity, can improve the situation. An elegant way to alleviate the problem of reduced lifetime is continuous top-up injection. It has been tested in many laboratories and extensive studies were recently performed at the APS [12]. Such an operation mode could maintain a constant thermal load on beamline optics and storage ring vacuum systems and could have the benefit of constant signal to noise ratio for the experiments. Nevertheless, a basic good lifetime before top-up should be envisaged in order to reduce radiation safety problems caused by high particle losses. Solving the lifetime problem by top-up injection puts higher requirements on the injection system. Reliability is of paramount importance; low power consumption and small beam dimensions for a loss free injection would be highly desirable. The SLS booster for example [13] is placed inside the storage ring tunnel. The large circumference results in many small little magnets with modest field. This provides a low power consumption $(230 \mathrm{~kW}$ for $3 \mathrm{~Hz}$ and correspondingly lower for less frequent top-up) and a small emittance $(7 \mathrm{~nm})$ for effective injection. A voltage and current controlled switch mode power supply allows a flexible adjustment of the ramp. The utilization of higher harmonics, on the other side, requires perfect beam conditions. Especially energy blow up due to instabilities must be avoided. Longitudinal coupled-bunch instabilities must be perfectly compensated. A series of measures were developed which are ranging from measures at the source, like mode shifting by temperature tuning or HOM plungers, damping of individual modes, superconducting zero-mode cavities, to broad-band feedback systems. All of them have been proved to work satisfactory. The new upcoming generations, are well advised to keep these most harmful instability effects well under control.

\subsection{STABILITY}

When beam sizes becomes smaller and smaller, the stability of the electron beam becomes more and more important. Measures for stabilization start with a proper site selection and the construction of the foundation for the storage ring-tunnel. All potential vibration sources should be removed from the nearby areas of the storage ring. Whatever has to be close, must be provided with proper damping features. A crucial issue is the temperature stabilization of the tunnel to avoid thermal movement. Synchrotron radiation must be absorbed in an antechamber on lumped absorbers equipped with powerful pumps under neat. In order to allow for a compensation of the residual movement with an orbit-feedback system, a fast and accurate beam position monitor system becomes essential. For the SLS, a new development was started which combines these usually conflicting requirements. In order to be fast, multiplexing is avoided and each BPM is equipped with four detectors, one for each pick-up button. For the calibration of these devices a pilot signal is used, which gives the guaranty for a high precision reading of the orbit [14]. A precise adjustment of the individual quadrupoles is important in order to reach a small vertical emittance and to reduce orbit distortions before switching on correction dipoles. High precision can be reached by placing groups of elements on a girder. Random displacements of the girders are generating a far smaller amplification on the orbit distortion than individually supported quadrupoles. These positions of the girders can be controlled by hydrostatic systems with capacitive or ultrasonic pick-ups and optical systems. To control the horizontal position of the SLS girder, an optical system will be used [15].

\section{CONCLUSIONS}

In spite of the unbeatable brilliance of future linac based SASE-FEL sources, storage ring based light sources will still be of great importance in the future. Regarding intensity, brilliance beyond $10 \mathrm{keV}$ photon energies and stability they are even superior. Compared with the existing 3rd generation sources, great progress will be reached with the new upcoming generation, i.e. DIAMOND, SLS and SOLEIL. These are machines of medium energy range that enter the regime of hard X-rays. To reach this goal with low undulator gaps and the use of higher har- 
monics of the spectrum, special attention must be given to maintain the necessary high beam performance. Top-up injection must become routine operation for these machines. This sets more stringent requirements on the injection system regarding reliability and power consumption. The high standard in storage ring technology will guarantee that storage ring based light sources will become members of the 4 th generation family.

\section{ACKNOWLEDGMENTS}

I would like to thank C. J. Bocchetta, M. Pool and J. L. Laclare for helping me with information material. I am also grateful to my colleagues at the SLS for providing me with some graphs, A. Streun for the Touschek lifetime and T. Schmidt for the Brilliance. H. Weyer I would like to thank for carefully reading the manuscript and making several suggestions, and M. Bugmann for typing the manuscript.

\section{REFERENCES}

[1] 10th ICFA Beam Dynamics Panel Workshop on 4th Generation Light Sources, Grenoble, 1996.

[2] C. Pellegrini, 'Is the X-ray Source the 4th Generation Light Source?', EPAC, Stockholm, 1998.

[3] J. Rossbach et al, 'Interdependence of Parameters of an X-ray FEL', NIM A374, 1996.

[4] J. L. Laclare, 'Light Source Performance Achievements', EPAC, Stockholm, 1998.

[5] M. Sommer, 'Optimization of the Emittance of Electron Storage Rings', LAL/RT/83-15, 1983.

[6] L. Teng, Minimum Emittance Lattice for Synchrotron Radiation Rings', APS LS-17, 1985.

[7] G. Wüstefeld, 'The Minimum of the Natural Emittance in the Triple Bend Achromat', BESSY TB 108/97, 1987.

[8] R. P. Walker, 'Wigglers', Sincrotrone Trieste ST/M94-4, 1994.

[9] Conceptual Design Report of the $7 \mathrm{GeV}$ Advanced Photon Source, ANL-87-15, 1987.

[10] A. A. Zholents, M. S. Zolotorev, 'Femtosecond X-ray Pulses of Synchrotron Radiation, PRL, Volume 76, Number 6, 1996.

[11] M. Böge, P. Marchand, A. Streun, 'Beam Lifetime Studies for the SLS Storage Ring', this conference.

[12] L. Emery, M. Borland, 'Top-up Operation Experience at APS', this conference.

[13] C. Gough et. al., 'SLS Booster Synchrotron', EPAC, Stockholm, 1998.

[14]M. Dehler et. al., 'BPM System for the Swiss Light Source', this conference.

[15] V. Schlott, PSI Annual Report 1998. 